\title{
Selection of arbitrarily scaled cross-sections in bending stiffness design
}

\section{Pasini \\ D.J. Smith \\ S.C. Burgess}

Department of Mechanical Engineering, University of Bristol, Queen's Building, University Walk, Bristol BS8 1TR, UK

\begin{abstract}
Performance indices can be used to model the relative structural efficiency of different cross-sectional shapes. Performance indices have been previously defined mainly for structural cross-sections which are scaled proportionally in size. This paper extends the method of performance indices by allowing scaling of the cross sections in any direction. A novel feature of the method described in this paper is the inclusion of the space envelope as a design parameter. The first part of the paper gives a derivation of the general solution for the performance index. The second part presents a graphical selection procedure and discusses the efficiency limits of cross-sections due to buckling instability. It concludes with a case study to demonstrate the method.
\end{abstract}

Keywords: Optimal shape, performance index, arbitrary scaling, constrained design, structural efficiency.

\section{NOTATION}

$\begin{array}{ll}A & \text { cross sectional area }\left(\mathrm{m}^{2}\right) \\ B & \text { width }(\mathrm{m}) \\ b & \text { internal width }(\mathrm{m}) \\ c_{1} & \text { constant for stiffness depending on boundary conditions and load } \\ D & \text { cross-section envelope dimensions }(b, h) \\ E & \text { Young's modulus }(\mathrm{GPa}) \\ F & \text { functional requirements } \\ h & \text { internal height }(\mathrm{m}) \\ H & \text { height }(\mathrm{m}) \\ I & \text { second moment of area }\left(\mathrm{m}^{4}\right) \\ k & \text { linear stiffness requirement }(\mathrm{N} / \mathrm{m}) \\ L & \text { length }(\mathrm{m}) \\ m & \text { mass }(\mathrm{Mg}) \\ M & \text { material parameters }\end{array}$


$P \quad$ performance index

$q \quad$ power of the performance index

$r_{g} \quad$ radius of gyration (m)

$S \quad$ shape of the cross section

$u \quad$ scaling factor of the widths

$v \quad$ scaling factor of the heights

W $\quad \operatorname{load}(\mathrm{N})$

$z \quad$ constant for the second moment of area

$\rho \quad$ material density $\left(\mathrm{Mg} / \mathrm{m}^{3}\right)$

$\delta \quad$ deflection (m)

$\psi \quad$ geometric transformer

\section{INTRODUCTION}

\subsection{Background}

The selection of light structures is often one of the main targets in structural design. Minimising mass helps to achieve lower material cost and low environmental impact. Low mass is also important where structures are required to produce low inertial loads and high natural frequency.

Performance indices and parametric equations can be used to carry out a systematic comparison of massefficiency of different material and shapes. Performance indices also provide a means of gaining insight in structural design.

The selection of efficient material-shape combinations has been investigated by several different authors over the last four decades. In the 1960's, Cox [1] and Shanley [2] produced design charts to help the designer to select directly efficient structures. Caldwell and Woodhead [3] also proposed a graphical method in the 1970's, which allows different beams and trusses to be compared. The results of the above methods can be used for structural members of any size provided all dimensions (length, width, and thickness) remain proportional. In the 1980's, Parkhouse [4,5,6] investigated the complementarity of shape and material for a structure and introduced the concept of structuring as a material dilution. Structuring is the process of shaping material, where a dilution factor describes the geometrical property relationship between different cross-sections and their representative solid sections. In the 1990's, Ashby [7] developed a material index, which includes the contribution of shape to the performance of the structure. This index is applicable mainly to the efficiency of geometrically unconstrained structures where the cross-sections can be proportionally scaled. However, the effect of height and width constraints was discussed briefly for bending stiffness design [8]. In 1994 Birmingham [10] explored the interaction between material and form and presented a graphical method to select structures. In the late 1990's, Burgess [11] extended the theory of the shape factors to model the efficiency of height constrained structures. 
In an earlier paper [12], an investigation was carried out into the effect of geometrical constraints in material selection. It was shown that geometrical constraints can have an important effect on what is the best material for a particular application.

In the first part of this paper the method of performance indices is extended to structures whose generic crosssection is scaled in any arbitrary direction. Performance maps are also presented in order to support cross-section selection in stiffness design. The 'space envelope' is introduced as a design variable. Attention is focused on the space each cross-section requires when a design requirement has to be met. This feature is relevant where minimising space rather than mass is the design goal.

In the second section of the paper a complementary graphical procedure is presented. This method will be used to consider the effect of buckling instability on the selection of the best cross-sections.

Finally, the performance index method is applied to a design case study showing that geometric constraints can have an important effect on what is the best cross-section for a particular application.

\subsection{Factors affecting the selection of the best cross-sections and materials}

In structural design it is very common that designers have to take into account spatial limitations. For example, height and width constraints are shown in Figure 1(a) and (b). Height constraints are common in floor structures and width constraints are common in wall structural components. A slope constraint is shown in Figure 1(c). This type of constraint is quite common in highly integrated structures such as cars and aircraft. These constraints restrict the magnitude and direction of scaling of the cross-sections and thus affect the selection of the lightest structural member.

Figure 1 shows examples where geometric constraints limit one or both of the envelope dimensions of different cross-sections. In these cases the cross-sections are forced to fit within a limited space and then to be scaled in a certain direction. For example, a height constraint, Figure 1(a), forces the cross sections to be scaled horizontally. A width constraint, Figure 1(b), imposes a vertical scaling direction. Figure 1(c) illustrates the effect of a sloped constraint on both the height and width of the cross-sections.

In the next section we show how to compare different arbitrarily scaled cross-sections in pure bending stiffness design. The materials are assumed to be homogenous and isotropic with the Young's modulus $E$ being the same in both tension and compression. Only pure bending stiffness design is examined in this work.

\section{GEOMETRIC TRANSFORMERS AND SCALING FACTORS}

In this section we derive expressions to compare the geometrical properties of cross-sections which are arbitrarily scaled. We define a 'cross-section' as a 'shape' which fits inside a rectangular 'envelope'. When the envelope is scaled, the enclosed shape is also scaled. 
Figure 2 shows two cross-sections, where their shapes are different and the relative scaling of their envelopes is arbitrary. The square, where the envelope and the shape are the same, is chosen as a reference section. The reasons for the choice of a square as a reference section are:

- the square can be changed into different proportions along the width and the depth into a rectangle, which is the envelope of any generic shape.

- the square belongs to the class of the rectangular shape. Rectangles meet the stiffness requirement in less space than all other shapes.

The following definitions are used to compare cross-sections:

For a generic cross-section

$I \quad=$ second moment of area

$A \quad=$ area of shape

$r_{g} \quad=$ radius of gyration

For the shape envelope

$B, H \quad$ : width and height

$I_{D} \quad=$ second moment of area of shape envelope

$A_{D} \quad=$ area of shape envelope

For the reference cross-section

$B_{o}, H_{o}$ : width and height, where $B_{o}=H_{o}$

$I_{o} \quad=$ second moment of area of reference section

$A_{o} \quad=$ area of reference section

$r_{g o} \quad=$ radius of gyration

Note that for the reference cross-section

$I=I_{D o}=I_{o}$ and $A=A_{D o}=A_{o}$

Table 1 shows expressions for the area, the second moment of area and the radius of gyration of the most common cross-sections, where $H$ and $B$ are the dimensions of the envelope and $h$ and $b$ are the internal width and height.

Firstly, we introduce two dimensionless shape transformers, $\psi_{A}$ and $\psi_{I}$, which relate area and second moment of area of a generic cross-section and its envelope They describe the geometric properties of the shape and are defined as: 


$$
\left\{\begin{array}{l}
\psi_{A}=\frac{A}{A_{D}} \\
\psi_{I}=\frac{I}{I_{D}}
\end{array}\right.
$$

Expressions of $\psi_{I}$ and $\psi_{A}$ are given in Table 2. For a rectangle and then for the reference, these shape transformers are unity:

$$
\begin{aligned}
& A_{o}=A_{D o} \rightarrow \psi_{A}=1 \\
& I_{o}=I_{D o} \rightarrow \psi_{I}=1
\end{aligned}
$$

Lastly, two linear multiplicators, $u$ and $v$, which we called scaling factors, define the scaling of the reference envelope dimensions, $B_{o}$ and $H_{o}$, and the generic cross-section dimensions, $B$ and $H$ :

$$
\left\{\begin{array}{l}
u=\frac{B}{B_{o}} \\
v=\frac{H}{H_{0}}
\end{array}\right.
$$

In constrained height design, shown in Figure 1(a), $v=1$ and this corresponds to direction $\mathrm{X}$ in Figure 1(d). In constrained width design, shown in Figure 1(b), $u=1$ and this corresponds to direction Y in Figure 1(d). When there is uniform proportional scaling of the cross-section $u$ is equal to $v$ and this corresponds to direction $\mathrm{Z}$ in Figure 1(d).

The relations, in terms of area and second moment of area, between the reference section and the generic shape envelope are:

$$
\left\{\begin{array}{l}
\frac{A_{D}}{A_{o}}=u v \\
\frac{I_{D}}{I_{o}}=u v^{3}
\end{array}\right.
$$

and between the generic and the reference cross-section are:

$$
\left\{\begin{array}{l}
\frac{A}{A_{o}}=\frac{A}{A_{D}} \frac{A_{D}}{A_{o}}=\psi_{A} u v \\
\frac{I}{I_{o}}=\frac{I}{I_{D}} \frac{I_{D}}{I_{o}}=\psi_{I} u v^{3}
\end{array}\right.
$$


In the next section we will use these expressions to derive a relative performance index in any arbitrary direction.

\section{PERFORMANCE INDEX FOR CROSS SECTIONS SCALED IN ANY ARBITRARY DIRECTION}

\subsection{The performance index and the design parameters}

A performance index $P$ is defined as a measure of mass efficiency. In general, the performance of a cross-section is a function $f($ )of four parameters:

$P=f(F, D, S, M)$

where $F$ represents the functional requirements or design input, $D$ describes the dimensions (width, $B$, and height, $H$ ) of the cross-section envelope, $S$ is a description of the shape properties of the cross-section and $M$ describes the material properties.

In a previous work [12], we examined conditions where $M$ and $D$ are variable. In this paper we are interested in selection conditions where $D, S, M$ are variables, i.e. where:

$$
P=f(D, S) \text { with } F \text { and } M \text { fixed }
$$

and

$$
P=f(D, S, M) \text { with } F \text { fixed }
$$

This paper will show that generic geometric constraints, such as those shown in Figure 1(a), 1(b) and 1(c), limit the direction and the magnitude of the scaling that can be carried out on a reference cross-section.

\subsection{The objective function}

For a given material, cross-section and set of design requirements, expressions for the mass $m$ and the elastic bending stiffness $k$ of a structural component are:

$$
\begin{aligned}
& m=\rho A L \\
& k=c_{1} \frac{E I}{L^{3}}
\end{aligned}
$$


where $c_{l}$ is a constant depending on the boundary conditions, $\rho$ is the density, $A$ is the cross-sectional area, $L$ is the length of all the structural elements and $I$ is the second moment of area. The objective function is minimisation of the mass.

For two structural members of the same length, $L$, the ratio of their masses, $m$ (for the generic case) and $m_{o}$ (for the reference case) is

$\frac{m}{m_{o}}=\frac{\rho}{\rho_{o}} \frac{A}{A_{o}}=\frac{\rho}{\rho_{o}} \psi_{A} u v$

where equation (4) is used for the ratio of the cross-sectional areas.

As maximising the performance index minimises the mass, then the ratio of the performance indices of the generic cross-section relative to the reference is:

$\frac{P}{P_{o}}=\frac{m_{o}}{m}=\frac{\rho_{o}}{\rho} \frac{1}{\psi_{A} u v}$

\subsection{Performance index for height constraint}

For a height constraint, the performance index can be derived by replacing the free variable $u$ and $v$ with expressions in terms of the design requirement. For bending stiffness design, the generic and the reference crosssections are required to meet the same stiffness requirement. For a given beam length L, and using equation (7), then:

$E_{o} I_{o}=I E$

The ratio of the stiffness of the two cross-sections can also be stated in terms of multipliers, $u$ and $v$, and geometric transformer, $\psi_{I}$, so combining equations (10) with (4) gives:

$\frac{E_{o}}{E}=\frac{I}{I_{o}}=\psi_{I} u v^{3}$

When the height of the two cross-sections is constrained $v=1$, and $u$ from (11) is:

$u=\frac{I}{I_{o}} \frac{1}{\psi_{I}}=\frac{E_{o}}{E} \frac{1}{\psi_{I}}$

and from (9) the ratio of the performance indices is: 
$\frac{P}{P_{o}}=\frac{\rho_{o}}{\rho} \frac{E}{E_{o}} \frac{\psi_{I}}{\psi_{A}}$

In equation (13), the material property group, $E / \rho$, can be separated from the contribution of the shape properties, $\psi_{I} / \psi_{A}$. The ratio $E / \rho$ is consistent with previous results for a height constraint $[\mathbf{8}, \mathbf{1 2}, \mathbf{1 3}]$.

\subsection{Performance index for width constraint}

When the width is constrained $u=1$, and $v$ from (11) is given by:

$v=\left(\frac{I}{I_{o}} \frac{1}{\psi_{I}}\right)^{\frac{1}{3}}=\left(\frac{E_{o}}{E} \frac{1}{\psi_{I}}\right)^{\frac{1}{3}}$

and from (9) the ratio of the performance indices is:

$\frac{P}{P_{o}}=\frac{\rho_{o}}{\rho}\left(\frac{E}{E_{o}}\right)^{\frac{1}{3}}\left(\frac{\psi_{I}}{\psi_{A}}\right)^{\frac{1}{3}}$

In equation (15), the material property group, $E^{I / 3} / \rho$, can be divided from the shape property group, $\psi_{I}^{I / 3} / \psi_{A}$. The ratio $E^{1 / 3} / \rho$ is consistent with previous results for a width constraint $[8, \mathbf{1 2}, \mathbf{1 3}]$.

\subsection{General solution of the performance index for arbitrary scaling}

Now we seek a general solution where $\mathrm{u} \neq 1$ and $v \neq 1$. The ratio of the performance indices can be written as:

$\frac{P}{P_{o}}=\frac{\rho_{o}}{\rho}\left(\frac{E \psi_{I}}{E_{o}}\right)^{q} \frac{1}{\psi_{A}}$

where $q$ is an expression which is yet to be determined, but known to be $q=1$ for constrained height (section 3.3) and $q=1 / 3$ for constrained width (section 3.4 ).

For generic $u$ and $v$ we write: 
$u=\left(\frac{E_{o}}{E} \frac{1}{\psi_{I}}\right)^{\alpha}$
$v=\left(\frac{E_{o}}{E} \frac{1}{\psi_{I}}\right)^{\beta}$

and using the expressions (17) in equation (11) gives:

$u v^{3}=\left(\frac{E_{o}}{E} \frac{1}{\psi_{I}}\right)^{\alpha+3 \beta}$

with the condition

$\alpha+3 \beta=1$

From equation (17), the exponents $\alpha$ and $\beta$ are:

$\left\{\begin{array}{l}\alpha=\lg _{\left(\frac{E_{o}}{E} \frac{1}{\psi_{I}}\right)} u=\lg _{\left(u v^{3}\right)} u \\ \beta=\lg _{\left(\frac{E_{o}}{E} \frac{1}{\psi_{I}}\right)} v=\lg _{\left(u v^{3}\right)} v\end{array}\right.$

The ratio of the performance indices $\frac{P}{P_{o}}$ follows from equation (9) using equations (17) through to (19), so that:

$\frac{P}{P_{o}}=\frac{\rho_{o}}{\rho}\left(\frac{E}{E_{o}} \psi_{I}\right)^{q} \frac{1}{\psi_{A}}$

where $q$ :

$q=\alpha+3 \beta=\log _{\left(u v^{3}\right)} u v=\frac{\ln u v}{\ln u v^{3}}$

Equation (20) allows the relative performance index for arbitrarily scaled cross-sections of different shapes and materials to be compared. The material and shape properties can be separated into distinct groups according to the selection condition. 
The exponent $q$ represents a parameter that describes the scaling of the dimensions of the cross-sectional envelopes due to change of either shape or material or both. In particular it is a function of $u$ and $v$, which are the scaling factors of the width and the height of the cross-section envelopes. From equation (20), it is evident that, in bending stiffness design, the general solution of the structural performance index is function of material, shape and envelope dimensions.

In a previous paper [12], we analysed conditions where the shape was fixed and the variable was the material. In the next section we will consider the selection where the material is fixed.

\section{LIMITING SHAPE REGIMES}

In this section, the general solution, equation (20), is used to provide a performance map, which can help the designer in the selection of light cross-sections for any scaling condition. This is similar to the chart of limiting material regimes presented in our earlier paper [12].

In stiffness design, the performance index $P$ for cross-sections of the same material with arbitrary scaling of the envelope is expressed by:

$$
P=\frac{\psi_{I}^{q}}{\psi_{A}}
$$

In Figure 3, regions for the scaling parameter $q=f(u, v)$ have been plotted. Values of $q=1 / 3, q=1 / 2, q=1$ are for constrained width, proportional scaling and constrained height respectively.

Shapes with values of $\psi_{I}$ near to 1 , such as hollow rectangles, perform relatively better for high values of $q$. In contrast when $q$ approaches zero the value of the area, $\psi_{A}$ is more important in comparison to the value of the second moment of area, $\psi_{I}$. This confirms that the direction of scaling has a very important effect not only on the material selection but also on the shape selection.

While the performance index $E^{q} / \rho$ gives the selection of the best material properties for cross-sections with a prescribed shape, $\psi_{I}{ }^{q} / \psi_{A}$ governs the selection of the best shape properties for arbitrarily scaled cross-sections. This analogy exists because shape properties and material properties influence the stiffness of a structural member together with its dimensions. Therefore for a given stiffness requirement, section envelopes experience dimensional variations in accordance to their values of $\psi_{I}$, and/or $E$. For solid cross-sections, replacing the shape properties of a structure has an analogous effect on the envelope sizes as replacing the material properties.

Figure 3 shows two examples of arbitrarily scaled envelopes. If the reference structure A, for instance, has a cross-section of unit dimensions, and, according to the stiffness requirement, is rescaled so that point A of the shape envelope moves to point $\mathrm{A}^{\prime}$, then $1 / 3<q<1 / 2$. Distinct regions for other ranges of $q$ are shown. 
Examples of a full range of solutions for equation (22) are shown in Figure 4 for two solid cross-section: an ellipse and a rectangle. The performance index has been plotted as a function of the scaling parameter $q$ using values of $\psi_{I}$ and $\psi_{A}$ given in Table 2.

Since the rectangle is the envelope of any shape, the curve for $P$ as a function of the scaling parameter, $q$, in Figure 4 is a horizontal line with $P=1$ for all $q$. The intersection point of the curves for the rectangle and the ellipse represents a value of $q$ where both shapes perform equally. For value of the scaling parameter $q$ less than 0.457 , an elliptical cross-section provides better performance. This is vice-versa for $q$ greater than 0.457 .

The scaling parameter for the shapes is $q$ and variation in $v$ as a function of $u$ and $q$ can be found by inverting equation (21) so that:

$$
v=u^{\frac{(1-q)}{(3 q-1)}}
$$

Curves of special values of $q$ and for which the two shapes have the same performance index are plotted in Figure 5. In bending stiffness design whereas for $q<0.457$ elliptical cross sections are lighter than rectangular crosssection and the rectangle is lighter for $q>0.457$.

In a later section we show an example of a design application where the limiting shape regimes shown in Figure 5 are used to compare the rectangle with I-sections.

\section{GRAPHICAL METHOD OF SELECTION}

This section presents a method of graphical selection which complements the performance chart shown in Figure 5. The general solution for arbitrary scaling allows the relative performance for cross-sections where either material or shape or both are variable. However, it does not provide a selection for the performance cross-sections where the only variable is the size of the envelopes.

The aim of this section is to provide a method to evaluate the efficiency of sections, which belong to the same class of shapes. To provide a solution, we demonstrate a graphical method that allows the selection of cross-sections where only the envelope dimensions vary. We start by considering solid cross-sections and then consider cases for hollow cross-sections.

\subsection{Solid cross-sections}

In bending stiffness design, the mass and the stiffness of a structural member are given by equations (7) and (8). Using the shape transformers given by the expressions (1) in equations (7) and (8) gives respectively the following equations for the mass and the stiffness: 
$k=\frac{c_{1}}{12 L^{3}} E \psi_{I} B H^{3}$

The performance criterion is given by the ratio of equations (25) and (24) and takes the form:

$$
\frac{k}{m}=\underbrace{\frac{c_{1}}{12 L^{4}}}_{\text {const }} \underbrace{\frac{E}{\rho}}_{\text {Material Shape }} \underbrace{\frac{\psi_{I}}{\psi_{A}}}_{\text {Envelope }} \underbrace{H^{2}}
$$

Equation (24) is divided in four groups. The first collects constant parameters, the second the material properties, the third the properties of the shape described by the shape transformers and the fourth is the envelope contribution which is a function $H=f(M, S)$ of material and shape.

Now, we consider structural members of the same material, length and subjected to the same boundary conditions. Using the shape transformer given in Table 2, the curves of performance $P=f(H)$ given by equation (24) are illustrated on the left of Figure 6 for solid cross-sections with $E=79 \mathrm{GPa}$ and $\rho=2.9 \mathrm{Mg} / \mathrm{m}^{3}$. Also illustrated on the right are curves of $H=g(B)$ with a constant stiffness $k$, for each solid cross-section obtained using equation (25) and Table 2. For a given stiffness requirement, the only possible scaling of the cross-section is along the curve of each shape.

Amongst all the cross sections, shown in Figure 6, the rectangle is the shape that occupies less space and therefore it provides a spatial benefit. No other shape can meet the stiffness requirement for the same dimensions (width and height) of the envelope. If the design goal is to minimize space rather than mass, then the best shape is the rectangle.

The performance of cross-sections is generally dependent on the geometric dimensions of the envelope. For two different envelopes, shown in Figure 6, one for a rectangle and the other for an ellipse, the performance of the ellipse is better than for the rectangle.

\subsection{Hollow cross-sections}

We now extend an earlier result for solid cross-sections to consider a hollow rectangle and a hollow ellipse.

As with Figure 6, Figure 7 shows curves of $H=f(B)$ with a constant stiffness (equation 7) and curves of performance $P=f(H)$ for both solid and hollow cross-sections (equations 24) with $E=79 \mathrm{Gpa}$ and $\rho=2.9 \mathrm{Mg} / \mathrm{m}^{3}$. With an increase of $b / B$ and $h / H\left(0<\frac{b}{B}<1,0<\frac{h}{H}<1\right)$, stiffness curves of hollow cross-sections move from curves provided by their respective solid shape $\left(\frac{b}{B}=0, \frac{h}{H}=0\right)$ upwards to a theoretical limit where $h=H$ and/or $b=B$ 
and the thickness' reduce to 0 . In Figure 7, we choose to plot a practical limit of the stiffness curves where $\frac{b}{B}=95 \%, \frac{h}{H}=95 \%$. Furthermore, all the performance index curves are in the range defined by the limit values $\frac{b}{B}=0, \frac{h}{H}=0$ and $\frac{b}{B}=100 \%, \frac{h}{H}=100 \%$.

The limiting curves, shown in Figure 7, can help to assess the performance of hollow cross-section whose envelopes are arbitrarily scaled.

\section{EFFICIENCY LIMITS}

Manufacturing constraints and mechanical buckling instability impose limits on the efficiency of sections. In this section, we consider a limit given through failure by buckling that occurs before the full plastic moment is reached. This is particularly the case for compression of a simple thin plate or flange or part of the web of a hollow section.

The material limits on the performance can be derived from the Euler's formula. For a given length, $L$, the limit to the performance of structures subjected to the same support conditions, is given by:

$$
r_{g}^{2}=\frac{I}{A}=\frac{\sigma_{y}}{E}\left(\frac{n L}{\pi}\right)^{2}
$$

where $\sigma_{y}$ is the yield stress and $n$ is the end support condition parameter.

Using the definitions (1) for the shape transformers with $A_{D}=B H$ and $I_{D}=1 / 12 B H^{3}$ to replace I and A and rearranging equation (27), gives:

$$
\frac{\psi_{I}}{\psi_{A}} \frac{H^{2}}{12}=\frac{\sigma_{y}}{E}\left(\frac{n L}{\pi}\right)^{2}
$$

Deriving $H$ in equation (28), gives the maximum height, $H_{l i m}$, of the envelopes of the cross-sections which will not buckle:

$$
H_{\lim }=\frac{n L \sqrt{12}}{\pi}\left(\frac{\sigma_{y}}{E}\right)^{0.5}\left(\frac{\psi_{A}}{\psi_{I}}\right)^{0.5}
$$

Figure 8 illustrates an example where the effect of buckling limits the range of the selectable sections for aluminium $\left(\sigma_{y}=500 \mathrm{MPa}, E=79, \rho=2.9 \mathrm{Mg} / \mathrm{m}^{3}\right)$ and steel $\left(\sigma_{y}=1600 \mathrm{MPa}, E=210, \rho=7,9 \mathrm{Mg} / \mathrm{m}^{3}\right)$ rectangular cross- 
sections. The dashed part of the curves of $P=f(H)$ and $H=g(B)$ represent structures that will buckle. Limiting values of the height limit, $H_{\text {lim }}$, are shown in Figure 8.

\section{DESIGN CASE STUDY}

In this section, the analysis presented in section 3 is used in a case study of a cantilever beam subjected to an end load.

The example is a 4-metre cantilever that must support an end load of $1000 \mathrm{~N}$ with an allowable end deflection, $\delta$, of $3.2 \mathrm{~cm}$. This is illustrated in Figure 9.

We consider an aluminium solid rectangular cross-section and an I-section since these are common crosssectional shapes. In the analysis the Young's Modulus, $E$, is $79 \mathrm{GPa}$ and the density, $\rho$, is $2.9 \mathrm{Mg} / \mathrm{m}^{3}$. We examine two constraints which lie on the aluminium square section, which is chosen as reference. First where there is steep slope. This corresponds to a value of $q=-0.21$. The second is a shallow constraint where there is a severe restriction on height but less restriction on width. Here $q=4.81$.

The limiting regimes for the two cross-sections and two design constraints are illustrated in Figures 10 and 11, where all regions in grey indicate that all I-sections provide the lightest cross-sections, while in the narrow white region only solid rectangle cross-sections give the lightest sections.

When there are narrow limits, i.e. $q=-0.21$ (steep slope), Figure 10 illustrates there are four solutions R1, R2, I1 and I2, that lie on the constraint line, where the curves for a prescribed stiffness intersect the constraint line. Points I1 and I2 lie in the regions where the I-sections provide the best performance compared to R1.

In the second constraint case, Figures 11 shows four solutions R1, R2, I1 and I2. Whereas point I1 lies in the region where the I-sections are lighter, I2 is located where the rectangle, R1, provides the best performance.

In Tables 3 and 4, numerical calculations validate the results.

\section{CONCLUDING REMARKS}

Previous research on material-shape selection produced solutions only for proportional scaling and constrained height. This paper has extended the method of performance indices by presenting a general solution for arbitrary scaling. This general solution allows selection of material and cross-sectional shape for a wide range of geometrical constraints.

We presented two complimentary methods for selecting light cross-sections in bending stiffness design. The first procedure generalises the approach of the performance index for arbitrarily scaled cross-sections different in shape and material. According to different constraint values of $q$ for two materials and cross-sections were presented for the performance index. A performance map illustrating limiting regimes where one cross-sectional shape performs 
better than other, was developed. The second method was a graphical solution that considers arbitrary scaling of the same cross-section. This method has been used also to investigate efficiency limits due to buckling instability.

The method presented in this paper allows the systematic comparison of the mass-efficiency of cross-section with different material and shapes properties. The method also provides a means of gaining insight in structural design.

An example of a cantilever subjected to an end load is presented. Different constraints on the cross-sections effect the results. One important finding is that a rectangular section can be lighter than an I-section.

\section{REFERENCES}

1. Cox, H.L., The design of structures of least weight - Oxford : Pergamon Press 1965.

2. Shanley, F. R., Weight-strength Analysis of Aircraft Structures, $2^{\text {nd }}$ edn, 1960 - (New York, Dover ).

3. Caldwell J.B. and Woodhead R.G., Ship structures: some possibilities for improvement, North East Cost Institution - Institution Engineers \& shipbuilders - Transaction - 1973, vol 89, 101 - 120.

4. Parkhouse, J.G, Structuring a process of Material Dilution, 1984, Proc. $3^{\text {rd }}$ Int. Conf. On Space Structures, H. Nooshin. Elsevier Applied Science Publishers, 367374.

5. Parkhouse, J.G. and Sepangi H.R., Macromaterials, 1993, in Building the future, ed. Garas, Armer and Clarke, E \& F.N. Spon, London, 3-13.

6. Parkhouse, J.G., Damage accumulation in structures, 1987, Reliability Engineering, Elsevier Applied Science Publishers Ltd , 17, 97-109

7. Ashby, M. F., Materials and shape. Acta Metall. Mater., 1991. 39(6), pp.1025 - 1039.

8. Ashby, M. F., Materials Selection in Mechanical Design, $1^{\text {st }}$ edn, 1992, (Pergamon Press, Oxford).

9. Ashby, M. F., Materials Selection in Mechanical Design, $2^{\text {nd }}$ edn, 1999, (Pergamon Press, Oxford).

10. Birmingham, R. W., A graphical exploration of the interaction between material and form in structural design, Doctoral thesis, University of New Castle upon Tyne, 1994.

11. Burgess, S. C, Shape factors and material indices for dimensionally constrained structures. Part1: beams. Proc. Instn Mech. Engrs, Part C, Journal of Mechanical Engineering Science, 1998, 212 (C2), pp 129-140.

12. Pasini, D., Burgess, S. C., Smith D.J, 2001, Performance indices for arbitrarily scaled rectangular cross-sections in bending stiffness design. Proc. Instn Mech. Engrs, Part L, Journal of Design and application, vol. 216. 1-13.

13. Charles, J.A., Crane, F.A.A., Selection and Use of Engineering Materials, $2^{\text {nd }}$ edn, 1989 (ButterworthHeinemann, Oxford).

14. Weaver, P. and Ashby, M. F., Material limits for shape efficiency. Journal of Engineering design, 1996, 7(2), pp.129-150.

15. Weaver, P. and Ashby, M. F., The Optimal Selection of Material and Section-Shape. Progress in Material Science, 1997, 41, pp.61-128. 